tribution due to spin-orbit coupling. The tendency to greater than linear behavior at higher fields is due to the variation of $\rho_{11}(B)$ in the denominator of $(2)$ and is thus an artifact of the inversion between $\rho_{21}(B)$ and $\sigma_{21}(B)$. For alloys less concentrated than $\sim 5$ at. $\% \mathrm{Fe}$ the uncertainty in the extrapolation is large, giving a less accurate $\Delta \sigma_{21}$.

${ }^{11}$ S. P. McAlister, L. R. Lupton, and C. M. Hurd, Solid State Commun. 25, 903 (1978).

${ }^{12}$ A. Fert and O. Jaoul, Phys. Rev. Lett. 28, 303 (1972).

${ }^{13} \mathrm{R}$. Huguenin and D. Rivier, Helv. Phys. Acta 38 , 900 (1965)

${ }^{14}$ A. Friederich, A. Fert, and J. Sierro, Solid State Commun. 13, 997 (1973).

${ }^{15}$ A. P. Murani, Phys. Rev. Lett. 37, 450 (1976), and J. Magn. Magn. Mat. $\underline{5}, 95$ (1977), and J. Phys. F $\underline{4}$, 757 (1974).

${ }^{16}$ P。A. Beck, J. Less-Common Met. 28, 193 (1972).

${ }^{17} \mathrm{R}$. Asomoza, thesis, Université de Paris-sud, 1975 (unpublished).

${ }^{18}$ The sign of the side-jump and skew contributions can be different since asymmetric skew terms appear in lowest order only in the second Born approximation (Ref. 3), whereas the side-jump terms appear in the first (Ref. 5). Thus if we take a simple scattering potential of the form $V+\lambda \vec{I} \cdot \vec{\sigma}$, where $V$ represents the upset in the host's periodic potential and $\lambda$ is the coupling constant between the electron's orbital momentum $\vec{i}$ and the ion's spin $\vec{\sigma}$, the lowest-order terms in the second approximation are of the form $\sim \lambda V^{2}$ for $\lambda<<V$ (Appendix A of Ref. 3) while the first-order terms are of the form $\sim \lambda V$. Hence the sign of the sidejump term depends on that of $V$ whereas that of the skew term does not.

${ }^{19}$ R. W. Klaffky and R. V. Coleman, Phys. Rev. B $\underline{10}$, 2915 (1974).

${ }^{20}$ A. Hamzic, S. Senoussi, I. A. Campbell, and A. Fert, Solid State Commun. $\underline{26}, 617$ (1978).

\title{
Phonon-Induced Fine Structure of Excitons in Solid Nitrogen
}

\author{
E. Boursey and V. Chandrasekharan \\ Laboratoire des Interactions Moléculaires et Hautes Pressions, Centre National de la Recherche Scientifique, \\ Université Paris-Nord, F-93440 Villetaneuse, France \\ and \\ P. Gürtler \\ II. Institut für Experimentalphysik der Universität Hamburg, D-2000 Hamburg 50, Federal Republic of Germany \\ and \\ E. E. Koch \\ Deutsches Elektronen-Synchrotron DESY, D-2000 Hamburg 52, Federal Republic of Germany \\ and \\ P. Kunsch \\ Chemistry Department, Eidgenössische Technische Hochschule, CH-8092 Zürich, Switzerland \\ and

\begin{abstract}
V. Saile
Sektion Physik der Universität München, D-8000 München 40, Federal Republic of Germany
\end{abstract}

(Received 19 July 1978)

\begin{abstract}
Results of quantitative high-resolution absorption measurements in the range of the $w^{1} \Delta_{\mathrm{u}}-X^{1} \Sigma_{\mathrm{g}}{ }^{+}$and $a^{1} \Pi_{\mathrm{g}} \leftarrow X^{1} \Sigma_{\mathrm{g}}{ }^{+}$derived exciton progressions in solid $\mathrm{N}_{2}$ are reported. The newly observed detailed fine structure of the vibrational bands is analyzed in terms of a sharp zero-phonon line and phonon-assisted exciton transitions. A theoretical analysis based on a strong-exciton-phonon-coupling model leads to good agreement with the observed experimental line shape.
\end{abstract}

The electronic structure of simple molecular crystals such as solid nitrogen and carbon monoxide has attracted much interest because of their key role for a better understanding of ex- citon states in weakly bound van der Waals solids. The absorption spectra of solid $\mathrm{CO}$ and $\mathrm{N}_{2}$ provided the first examples for the perturbation of the excited electronic states of simple molecules by 
the crystal field. ${ }^{1,2}$ However, almost no quantitative information has been obtained experimentally on other important features of the absorption. In particular, the line shape of the bands has never been analyzed, although a detailed understanding of the coupling of exciton states to the crystal might be expected and a number of calculations and predictions for the exciton-phonon coupling in vibrating molecular crystals are available in the literature..$^{3-8}$

Recently, high-resolution low-temperature vacuum-ultraviolet spectroscopy ${ }^{9-11}$ has provided new ways for examining higher excited states of van der Waals solids, such as surface excitons in rare-gas solids ${ }^{11}$ and vibrational and librational excitations of matrix-isolated molecules. ${ }^{9}$ In the present work we apply these techniques to the investigation of exciton transitions in pure solid nitrogen. A careful examination and analysis of the exciton absorption line shapes for two weak transitions forming extended progressions in the spectral range from 8.5 to $12.5 \mathrm{eV}$ will be presented. On of the important results of our study is the detection of extremely sharp [full width at half maximum (FWHM) $\leqslant 1 \mathrm{meV}$ ] zero-phonon lines (ZPL) and accompanying phonon sidebands for each vibrational sub-band of an electronic excitation.

The experiments have been performed with two different monochromators. By using the 10-m spectrograph ${ }^{9}$ located at Meudon, France, we have recorded the spectra at a high resolution of
$0.014 \AA$ in first order. The spectrograph was illuminated with a BRV continuum source and calibrated with atomic emission lines. Data obtained with this instrument have been mainly used for establishing accurate energy positions of the bands. Quantitative intensity measurements have been performed at a resolution of $0.15 \AA$ with 3 $\mathrm{m}$ normal-incidence monochromator with synchrotron radiation from the DORIS storage ring at DESY, Germany. ${ }^{10}$ This instrument was calibrated by rare-gas absorption lines. Light from the exit slit traversed a film of solid $\mathrm{N}_{2}$ condensed on a $\mathrm{LiF}$ window, the temperature of which ranged from 6 to $35 \mathrm{~K}$. These experiments were carried out under ultrahigh-vacuum conditions $(p \simeq 5$ $\times 10^{-10}$ Torr). The transmitted light was detected by a sodium salycilate photomultiplier (EMI 9804) combination. The sample gas was nitrogen of A 52 grade from Air Liquide. Its purity was checked during condensation by a mass spectrometer. More details will be given in a forthcoming paper. ${ }^{12}$

A general view of the absorption of solid $\mathrm{N}_{2}$ in the range of the $a^{1} \Pi_{\mathrm{g}}-X^{1} \Sigma_{\mathrm{g}}{ }^{+}$and $w^{1} \Delta_{\mathrm{u}}-X^{1} \Sigma_{\mathrm{g}}{ }^{+}$ transitions is shown in Fig. 1. Whereas the $a$ system forms a progression of fairly broad bands, the stronger $w$ system forms a progression of comparatively sharp bands. Both transitions are electrically dipole forbidden in the gaseous phase. ${ }^{13}$ They become partly allowed in the lower symmetry of the excited molecular state in the crystal. The symmetry of the free $\mathrm{N}_{2}$ mol-

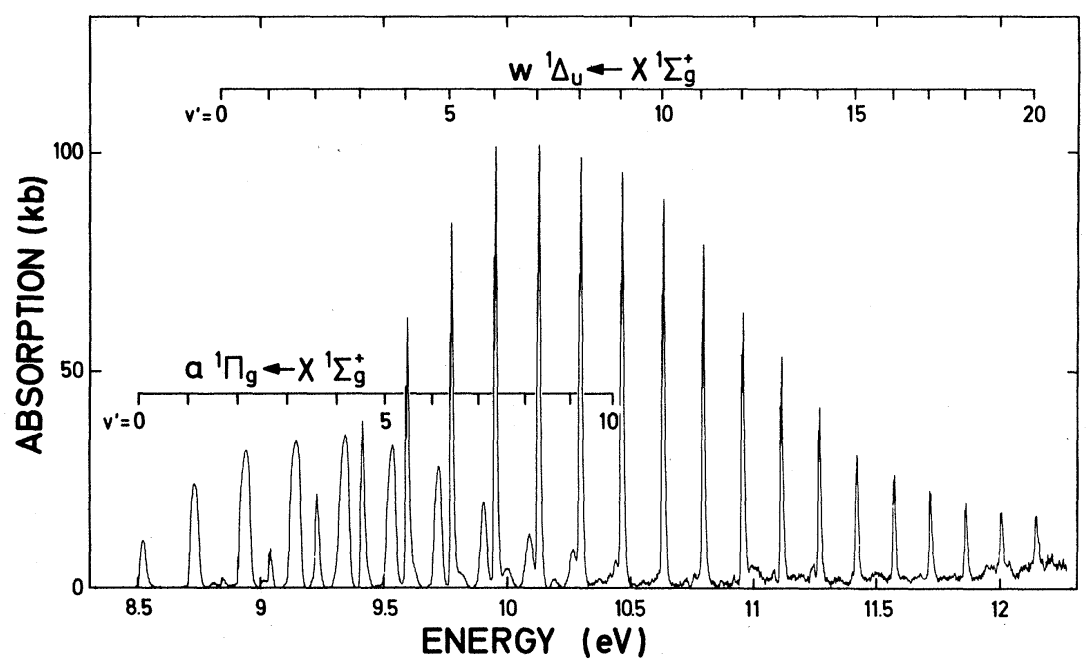

FIG. 1. Overview of the absorption spectrum from solid nitrogen in the photon energy range from 8.5 to $12.3 \mathrm{eV}$. The two progressions corresponding to the gas phase $a^{1} \Pi_{\mathrm{g}}-X^{1} \Sigma_{\mathrm{g}}{ }^{+}$and $w^{1} \Delta_{\mathrm{u}}-X^{1} \Sigma_{\mathrm{g}}{ }^{+}$transitions are labeled. Note that we have chosen the zero-phonon features at the low-energy sides of the bands as the origins. 
ecule $\left(D_{\infty h}\right)$ is reduced to the site symmetry group $\left(C_{3 i}\right)$ in the $\alpha$ phase of solid $\mathrm{N}_{2}$. (This is the low-temperature cubic phase for $T \leqslant 35.6$ $\mathrm{K} .{ }^{14}$ ) Thus one would expect an $E_{\mathrm{g}}-A_{\mathrm{g}}$ and an $E_{\mathrm{u}}-A_{\mathrm{g}}$ transition corresponding to the $a$ and $w$ systems, respectively. Consideration of the factor group $T_{h}$ of the molecular crystal yields a splitting of the $a^{1} \Pi_{\mathrm{g}}$ final state into one $E_{\mathrm{g}}$ and two $F_{\mathrm{g}}$ components and of the $w^{1} \Delta_{\mathrm{u}}$ state into one $E_{\mathrm{u}}$ and two $F_{\mathrm{u}}$ components. Consequently the $\mathrm{g}$ - $\mathrm{g}$ transitions remain electrically forbidden in the crystal, whereas transitions to $F_{\mathrm{u}}$ final states become allowed. This implies that the $w^{1} \Delta_{\mathrm{u}}-{ }^{1} \Sigma_{\mathrm{g}}{ }^{+}$ transition becomes dipole allowed, whereas the mechanism for the weaker $a^{1} \Pi-X^{1} \Sigma_{\mathrm{g}}{ }^{+}$transition is magnetic dipole and electric quadrupole as in the gas phase. We note, however, already at this point, that our results do not show a factor-group splitting. As can be seen from Fig. 1 and Table I, both progressions have an extremely small oscillator strength even in the crystal. Comparison of the constants derived from a polynomial fit to the progressions with those known for the corresponding transitions in the gaseous phase shows that both are very similar. Thus, e.g., the quanta of the intramolecular vibration for the $a$ system are $208 \mathrm{meV}$ (Ref. 13) and $205 \mathrm{meV}$ in the gaseous and solid phases, respectively. Solidification leads to an almost rigid shift of roughly $50 \mathrm{meV}$ of all bands towards lower energies.

Compared to previous results ${ }^{2}$ our data show a far more detailed fine structure of the various bands. As an example we show in Fig. 2 the range of the $v^{\prime}=0 \mathrm{~b}$ and of the $w^{1} \Delta_{\mathrm{u}}$ system and the $v^{\prime}=2$ band of the $a^{1} \Pi_{\mathrm{g}}$ system. Although both bands are quite different in their general appearance we note that they show as a common feature a very sharp line at the low-energy side followed by broader partly structured features towards

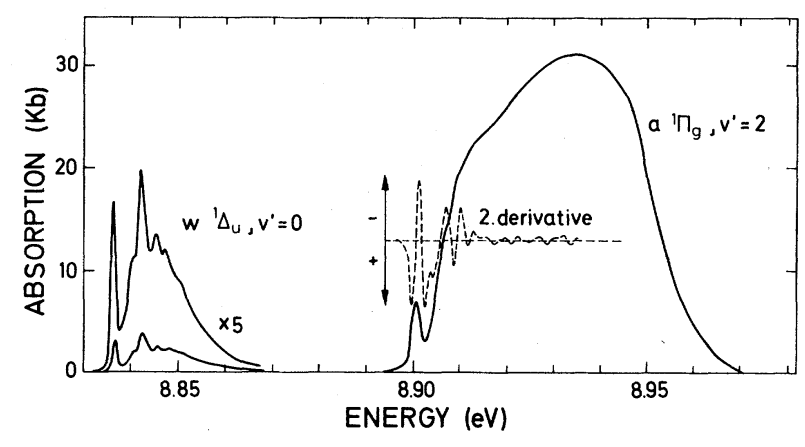

FIG. 2. Absorption spectrum of solid $\mathrm{N}_{2}$ at $6 \mathrm{~K}$ in the range 8.83 to $8.98 \mathrm{eV}$. For the $v^{\prime}=2$ band of the $a^{1} \pi_{\mathrm{g}}$ system the second derivative is also shown.

higher energies. As shown by the second derivative of the $v^{\prime}=2$ band these features are very similar for both systems as far as the spacings are concerned (see also Table I).

The splitting of the bands of the $a$ system has been discussed as a Davydov splitting by Brith and Schnepp. ${ }^{2}$ We reject this interpretation since the observed detailed structure with its constant splitting over the progression is not compatible with a simple Davydov splitting into two components. Instead of such an explanation, which is applicable in a weak-exciton-phonon-coupling case, we propose an analysis for the fine structure based on a polaron model ${ }^{5}$ with strong exciton-phonon coupling. ${ }^{3,5-7,15}$

Within this model we interpret the sharp maximum appearing on the low-energy side of each vibrational band as the ZPL. The electronic excitation leads to a strong localized deformation of the crystal. The features at higher energies correspond to the coupling of the electronic excitation to lattice modes in the deformed potential at this site. For the $v^{\prime}=0$ band of the $w$ system we can readily identify several phonon sidebands

TABLE I. Excitation energies (in eV) for the zero-phonon lines (ZPL) in solid $N_{2}$ for the $a^{1} \Pi_{\mathrm{g}}$ and the $w^{1} \Delta_{\mathrm{u}}$ progressions for $v^{\prime}<6$ and energetic positions of the phonon-induced fine structure with respect to the ZPL. For each vibrational band the oscillator strength $f$ is given. Positions of the ZPL for the $a$ system given in parentheses have been estimated.

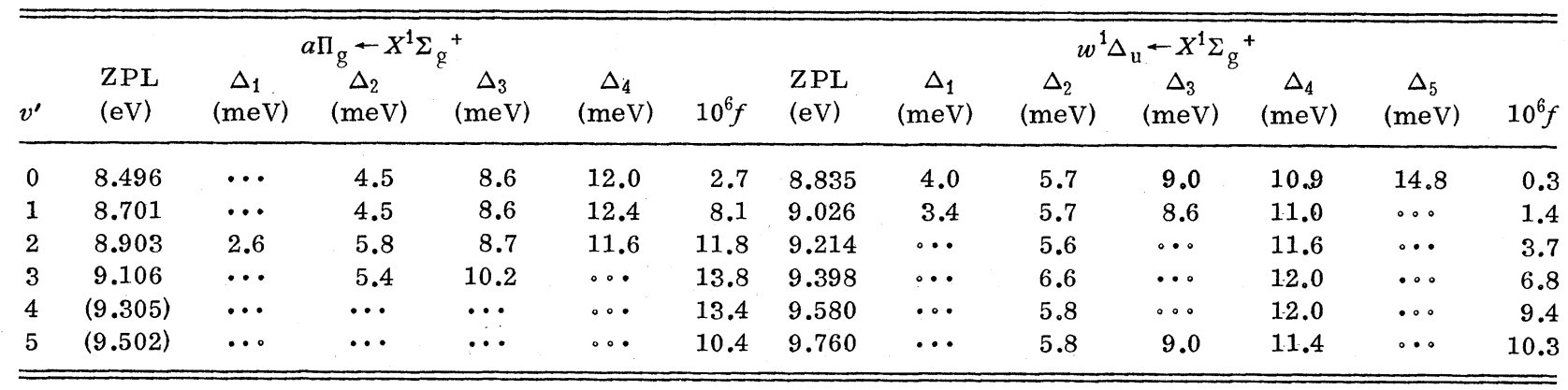


corresponding to the excitation of one or more phonons. Their energies are listed in Table I together with data for the higher vibrational bands of this as well as for the $a \Pi_{\mathrm{g}}$ progression.

To further substantiate this analysis we have performed calculations for 0-K temperature which are based on a local picture treating the lattice dynamics in a "supermolecule model,"8 in which only a few shells around the excited molecule are allowed to participate in the nuclear motions. In the calculations the following assumptions have been made ${ }^{16,17}$ : (i) The exciton-phonon coupling is linear in its external coordinates; (ii) the external oscillators are harmonic; and (iii) only one translational $\omega_{T}$ and one librational $\omega_{L}$ mode, both having g symmetry in the site group of the excited molecule ${ }^{8}$ are coupled to the exciton state. In order to simulate the experimental curve, the one-phonon shape was chosen to be doubly peaked at $\omega_{T}=5.9 \mathrm{meV}$ and $\omega_{L}=3.7$ $\mathrm{meV}$. These frequencies are close to the known $k=0$ values of the librational and the translational modes of the vibrating $\mathrm{N}_{2}$ crystal as observed by Raman and infrared spectroscopy. ${ }^{18,19}$ The higher phonon sideband was obtained by convoluting the one-phonon band $k$ times with itself and weighting with a Poisson distribution of the following form ${ }^{15-17}: P_{k}=S^{k} e^{S / k}$ !, where $S$ is the coupling strength ${ }^{15}$ and $e^{-S}$ represents the ratio of the ZPL intensity to the integrated intensity of the entire band. The result of this calculation with a value of $S=2.2$ is shown for the $v^{\prime}=0$ band of the $w$ system in Fig. 3. The agreement with

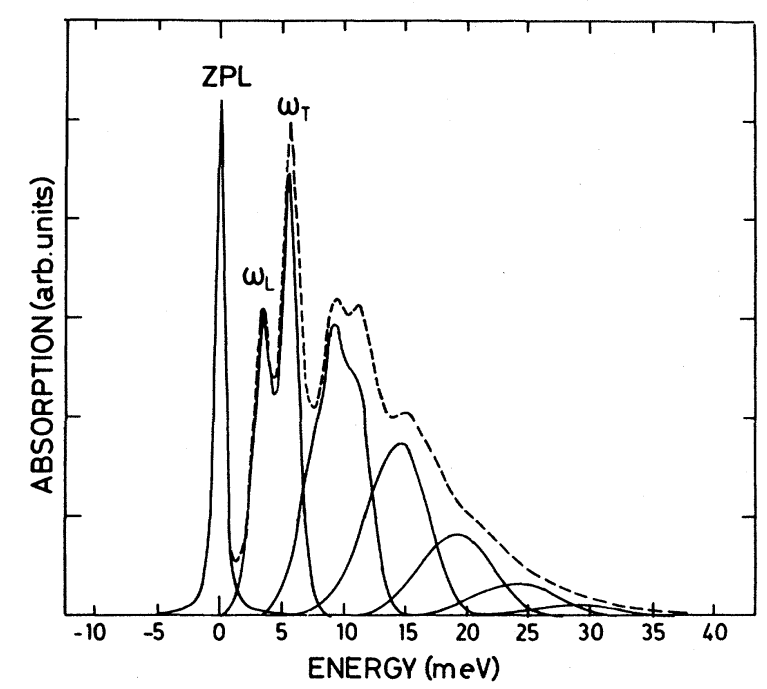

FIG. 3. Simulated line shape of the $v^{\prime}=0$ band of the $w^{1} \Delta_{\mathrm{u}}-X^{1} \Sigma_{\mathrm{g}}{ }^{+}$system at $T=0$ with a coupling strength of $S=2.2$. For details see text. the experimental results is very good, thus verifying cur analysis of the shoulder at around 4.0 meV above the ZPL as a librational mode of the excited $\mathrm{N}_{2}$ molecules and the mode with $\omega_{T} \approx 5.7$ $\mathrm{meV}$ as the translational mode of the vibrating $\mathrm{N}_{2}$ crystal. Although apparently the same modes are excited in the $w$ and in the $a$ systems (see Table I), the coupling strength for the $a$ system is much greater, leading to an enhancement of the multiphonon processes.

We would like to thank Professor O. Schnepp for a critical reading of the manuscript and valuable comments. This work has been supported in part by Bundesministerium für Forschung und Technologie (BM FT) from funds for synchrotron radiation research.

${ }^{1}$ K. Dressler, J. Quant. Spectrosc. Radiat. Transfer 2, 683 (1962).

${ }^{2}$ M. Brith and O. Schnepp, Mol. Phys. 9 , 473 (1965); see also J.-Y. Roncin and N. Damany, C. R. Acad. Sci. 260,6069 (1965).

${ }^{3}$ A. S. Davydov, Theory of Molecular Excitons (Plenum, New York, 1971).

${ }^{4}$ R. M. Hexter, J. Chem. Phys. 46, 2300 (1967).

${ }^{5}$ S. Fischer and S. A. Rice, J. Chem. Phys. 52, 2089 (1970).

${ }^{6}$ K. Cho and Y. Toyozawa, J. Phys. Soc. Jpn. $\underline{30}$, 1555 (1971).

${ }^{7}$ R. M. Hochstrasser and P. N. Prasad, J. Chem. Phys. 56, 2814 (1972).

${ }^{8}$ P. L. Kunsch and K. Dressler, J. Chem. Phys. $\underline{68}$, 2550 (1978); P. L. Kunsch, to be published.

${ }^{9}$ E. Boursey and J.-Y. Roncin, J. Mol. Spectrosc. $\underline{55}$, 31 (1975).

${ }^{10}$ V. Saile, P. Gurtler, E. E. Koch, A. Kozevnikov, M. Skibowski, and W. Steinmann, Appl. Opt. 15, 1559 (1976).

${ }^{11}$ V. Saile, M. Skibowski, W. Steinmann, P. Gưrtler, E. E. Koch, and A. Kozevnikov, Phys. Rev. Lett. $\underline{37}$, 305 (1976).

${ }^{12}$ P. Gurtler et al., to be published.

${ }^{13}$ Y. Tanaka, M. Ogawa, and A. S. Jursa, J. Chem.

Phys. 40, 3690 (1964).

${ }^{14} \mathrm{~J}$. A. Venables and C. A. English, Acta Crystallogr., Sect. B. 30, 929 (1974).

${ }^{15}$ D. B. Fitchen, in Physics of Color Centers, edited

by W. B. Fowler (Academic, New York, 1968).

${ }^{16}$ K. K. Rebane, Impurity Spectra of Solids (Plenum, New York, 1970).

${ }^{17}$ M. H. L. Pryce, in Phonons in Perfect Lattices and in Lattices with Point Imperfections, edited by R. W. H. Stevenson (Oliver and Bryd, Edinburgh, London, 1966), p. 403.

${ }^{18} \mathrm{~A}$. Ron and O. Schnepp, J. Chem. Phys. $\underline{46}, 3991$ (1967).

${ }^{19}$ A. Anderson, T. S. Sun, and M. C. A. Donkersloot, Can. J. Phys. $\underline{48}, 2265$ (1970). 